\title{
Resonant Generation of Topological Modes in Trapped Bose Gases
}

\author{
V.I. Yukalov ${ }^{1,2}$, K.-P. Marzlin ${ }^{3,2}$, and E.P. Yukalova ${ }^{4}$ \\ ${ }^{1}$ Bogolubov Laboratory of Theoretical Physics \\ Joint Institute for Nuclear Research, Dubna 141980, Russia \\ ${ }^{2}$ Fachbereich Physik, Universität Konstanz, Fach M 674 \\ D-78457 Konstanz, Germany \\ ${ }^{3}$ Department of Physics and Astronomy \\ 2500 University Drive NW, Calgary \\ Alberta T2N 1N4, Canada \\ ${ }^{4}$ Department of Computational Physics \\ Laboratory of Information Technologies \\ Joint Institute for Nuclear Research, Dubna 141980, Russia
}

\begin{abstract}
Trapped Bose atoms cooled down to temperatures below the Bose-Einstein condensation temperature are considered. Stationary solutions to the Gross-Pitaevskii equation (GPE) define the topological coherent modes, representing nongroundstate Bose-Einstein condensates. These modes can be generated by means of alternating fields whose frequencies are in resonance with the transition frequencies between two collective energy levels corresponding to two different topological modes. The theory of resonant generation of these modes is generalized in several aspects: Multiple-mode formation is described; a shape-conservation criterion is derived, imposing restrictions on the admissible spatial dependence of resonant fields; evolution equations for the case of three coherent modes are investigated; the complete stability analysis is accomplished; the effects of harmonic generation and parametric conversion for the topological coherent modes are predicted. All considerations are realized both by employing approximate analytical methods as well as by numerically solving the GPE. Numerical solutions confirm all conclusions following from analytical methods.
\end{abstract}

PACS numbers: 03.75.-b, 03.75.Fi, 05.45.-a 


\section{Introduction}

Dilute Bose gases at low temperatures, when almost all atoms are in Bose-Einstein condensate, are well described by the GPE (see reviews [1]-[5]). Since the latter is a type of the nonlinear Schrödinger equation, it should possess the whole spectrum of stationary solutions. In the presence of a trapping potential, the related energy spectrum is, in general, discrete. Stationary solutions to the GPE are, by definition, the topological coherent modes, whose ground state describes the standard Bose-Einstein condensate, while the higher states correspond to nonground-state Bose-Einstein condensates [6].

It is worth saying a few words recalling where the name topological coherent modes comes from. Different stationary solutions to the GPE, associated with different energy levels, display principally different spatial shapes, in particular, different number of zeros. Because of their distinct spatial topology, the modes are termed topological. These should not be confused with elementary collective excitations, defined by linear Bogolubov De Gennes equations. Such elementary excitations describe small oscillations around a given stationary solution and do not change the topology of the latter. Since the GPE is nonlinear, its solutions can also be named nonlinear modes, which would stress their principal difference from elementary excitations satisfying the linear Bogolubov - De Gennes equations. However, the elementary excitations, produced by strong perturbations, are sometimes also called nonlinear. Therefore the term topological, characterizing dissimilar modes, seems to be more precise. The topological modes are specified as coherent due to the fact that the GPE can be interpreted as an exact equation for coherent states [7, 8].

The possibility of resonant generation of arbitrary topological coherent modes was advanced in Ref. 6]. A particular case of vortex creation was suggested in [9, 10, 11]. The properties of such modes were also studied in [12] - 22] and a dipole topological mode was excited in experiment [23. Bose-Einstein condensates with topological coherent modes exhibit a variety of interesting features which could find many applications. Among these features, we could mention the following. Mode locking: this is the effect under which the fractional mode populations are locked to stay in the vicinity of their initial values 6. 8, 22. Mathematically, this effect is analogous to self-trapping occurring for atoms in double-well potentials [24, 25, 26]. Critical dynamics: an abrupt qualitative change of dynamics of fractional mode populations under an infinitesimally small variation of the pumping parameters [8, 17, 21, 22. A mathematically similar effect in the case of double-well potentials is the dynamic phase transition between the Rabi and Josephson regimes [24, 25, 27]. Interference patterns: specific interference fringes arising because of differing spatial shapes of different topological modes [8, 21. Interference current: fastly oscillating current, existing even inside a single-well trap [8, 21]. Such a type of current, arising between two interpenetrating populations, not separated by any barrier, is, on occasion, called the internal Josephson effect [28, 29]. Atomic squeezing: narrowing of the dispersion corresponding to the mode population difference, compared to the dispersion related to dipole transitions [8]. A similar feature is illustrated by two-component condensates [30]. Irreducible modes: these are the topological modes that have no linear counterparts, so that they cannot be considered as analytical continuations, under increasing of nonlinearity, of the related linear modes [18, 19]. Such modes, in addition to strong nonlinearity, require the presence of multiwell potentials [31, 32, 33, 34. An 
investigation of the mode spectrum in the case of single-well traps shows that in this case nonlinear modes are reducible and can be treated as analytical continuations of linear counterparts [6, 8, 15, 35, 36].

In the present paper, we generalize the theory of resonant formation of topological coherent modes and study the features that have not been considered in previous publications. The most important novel points are as follows:

1. The possibility of resonant generation of multiple topological coherent modes is described. This can be achieved by subjecting a trapped Bose-Einstein condensate to the action of several alternating fields, whose frequencies are tuned to distinct transition frequencies related to different modes (Sec. 21).

2. A general criterion is derived, showing when nonlinear modes cannot be generated even if the applied alternating field is in perfect resonance with the corresponding transition frequency. This condition relates the spatial dependence of the trapping potential and that of the alternating field (Sec. 3).

3. Simultaneous generation of two excited coherent modes is studied in detail. The resulting dynamical system describes three coexisting nonlinear modes (Sec. 4).

4. The phase portrait of the three-mode dynamical system is investigated. All fixed points are found and their stability is analysed (Sec. 6).

5. Harmonic generation of topological modes is shown to exist. This effect is analogous to optical harmonic generation (Sec. 7 ).

6. Parametric conversion for topological modes is another effect having its optical counterpart. To realize this effect, it is necessary to subject trapped atoms to the action of several alternating fields (Sec. 7).

7. The principal feature of this article is that all consideration has been done in two ways, by applying approximate analytical methods and also by numerically solving the GPE. Numerical solutions confirm all effects described analytically.

\section{Resonant Generation of Multiple Modes}

Dilute Bose-condensed gases at low temperature are characterized [1] - [5] by a coherent field $\varphi(\mathbf{r}, t)$, which is a wave function satisfying the GPE

$$
i \hbar \frac{\partial}{\partial t} \varphi(\mathbf{r}, t)=(\hat{H}[\varphi]+\hat{V}) \varphi(\mathbf{r}, t)
$$

where the nonlinear Hamiltonian

$$
\hat{H}[\varphi]=-\hbar^{2} \frac{\nabla^{2}}{2 m_{0}}+U(\mathbf{r})+N A_{s}|\varphi(\mathbf{r}, t)|^{2}
$$


contains a trapping potential $U(\mathbf{r})$ and the interaction intensity

$$
A_{s} \equiv 4 \pi \hbar^{2} \frac{a_{s}}{m_{0}}
$$

with $m_{0}$ being atomic mass; $a_{s}$, scattering length; and $N$, the total number of atoms. The wave function is normalized to unity, so that $\|\varphi\|=1$. The confining potential can be modulated by applying an additional field $\hat{V}=V(\mathbf{r}, t)$.

The topological coherent modes are the solutions to the stationary GPE

$$
\hat{H}\left[\varphi_{n}\right] \varphi_{n}(\mathbf{r})=E_{n} \varphi_{n}(\mathbf{r})
$$

where $n$ is a labelling multi-index. The transition frequencies for two distinct modes $m \neq n$ are

$$
\omega_{m n} \equiv \frac{1}{\hbar}\left(E_{m}-E_{n}\right)
$$

The trap modulation is resonant if the frequency of an applied alternating field is tuned close to one of the transition frequencies (5). This resonant field can induce transitions between the considered modes. Everywhere in what follows, talking about resonance, we keep in mind the resonant transitions between distinct topological modes. This should not be confused with parametric resonance, when one considers a single mode, whose width can become divergent under special conditions on the amplitude of a perturbing field 37.

Previously, the resonant excitation of topological modes has been considered for the case of a sole resonant field coupling two chosen modes [6, 8]. Now, we turn to the most general case, when there are several alternating fields, so that the modulating potential is a sum

$$
V(\mathbf{r}, t)=\sum_{j}\left[V_{j}(\mathbf{r}) \cos \left(\omega_{j} t\right)+V_{j}^{\prime}(\mathbf{r}) \sin \left(\omega_{j} t\right)\right]
$$

of fields with different frequencies $\omega_{j}$. Generally, the amplitudes $V_{j}(\mathbf{r})$ and $V_{j}^{\prime}(\mathbf{r})$ can also be slow functions of time, whose temporal variation is slow compared to that of $\cos \left(\omega_{j} t\right)$, such that

$$
\frac{1}{\hbar \omega_{j}}\left|\frac{\partial V_{j}}{\partial t}\right| \ll 1, \quad \frac{1}{\hbar \omega_{j}}\left|\frac{\partial V_{j}^{\prime}}{\partial t}\right| \ll 1 .
$$

If these amplitudes are such slow functions of time, then, varying them adiabatically, one could induce the Landau-Zener tunneling [31, 33]. But here we shall consider only the transitions caused by the fastly oscillating $\cos \left(\omega_{j} t\right)$. Each frequency $\omega_{j}$ is assumed to be close to one of the transition frequencies (5), so that the resonance conditions

$$
\left|\frac{\Delta_{m n}}{\omega_{m n}}\right| \ll 1, \quad \Delta_{m n} \equiv \omega_{j}-\omega_{m n},
$$

are valid.

The modulating potential (6) can be presented as

$$
V(\mathbf{r}, t)=\frac{1}{2} \sum_{j}\left[B_{j}(\mathbf{r}) e^{i \omega_{j} t}+B_{j}^{*}(\mathbf{r}) e^{-i \omega_{j} t}\right],
$$


with

$$
B_{j}(\mathbf{r}) \equiv V_{j}(\mathbf{r})-i V_{j}^{\prime}(\mathbf{r}) .
$$

The modulation can be realized by varying the trapping magnetic fields, by invoking laser spoons, or resorting to other means [6, 8, 9, 10, 11].

There exist two characteristic quantities, called transition amplitudes; one is the matrix element

$$
\alpha_{m n} \equiv N \frac{A_{s}}{\hbar}\left(\left|\varphi_{m}\right|^{2}, 2\left|\varphi_{n}\right|^{2}-\left|\varphi_{m}\right|^{2}\right),
$$

due to the interatomic interactions (31), and another one is the matrix element

$$
\beta_{m n} \equiv \frac{1}{\hbar}\left(\varphi_{m}, \hat{B}_{j} \varphi_{n}\right)
$$

related to the amplitude $\hat{B}_{j}=B_{j}(\mathbf{r})$ of the modulating potential (9). Here, (.,.) denotes the usual scalar product of Schrödinger theory. To avoid intensive power broadening, these amplitudes (11) and (12) have to satisfy the inequalities

$$
\left|\frac{\alpha_{m n}}{\omega_{m n}}\right| \ll 1, \quad\left|\frac{\beta_{m n}}{\omega_{m n}}\right| \ll 1,
$$

where $m \neq n$ and whose meaning was explained in detail in 8. Conditions (13) allow for an effective generation of nonlinear modes by resonant fields. The first of these restrictions, briefly speaking, can be reduced to the limitation on the number of atoms that can be transferred to an excited coherent mode [8]. This limiting number of atoms is close to the critical number of atoms with attractive interactions, for which the Bose-Einstein condensate preserves its stability [6, 8, 38, 39]. Therefore the resonant generation of nonlinear modes is feasible for atoms with positive as well as negative scattering lengths.

The topological coherent modes, being the solutions to the nonlinear eigenproblem (4), do not compulsory form a complete orthonormal basis. However, the modes $\varphi_{n}(\mathbf{r})$ can always be normalized, so that $\left\|\varphi_{n}\right\|=1$. And the set $\left\{\varphi_{n}(\mathbf{r})\right\}$ of all linearly independent functions forms a total basis [8], permitting one to look for the solution of the GrossPitaevskii equation (11) in terms of the presentation

$$
\varphi(\mathbf{r}, t)=\sum_{n} c_{n}(t) \varphi_{n}(\mathbf{r}) \exp \left(-\frac{i}{\hbar} E_{n} t\right),
$$

where $c_{n}(t)$ are unknown functions of time. Note that for some nonlinear eigenproblems, it has been rigorously proved [40, 41, 42] that the set of the corresponding eigenfunctions forms a complete basis. In our case, it is sufficient to require that the functions $c_{n}(t)$ are slowly varying, such that

$$
\frac{1}{\omega_{m n}}\left|\frac{d c_{n}}{d t}\right| \ll 1,
$$

and conditions (13) hold true. Then the expansion (14) is uniquely defined by means of the averaging technique [43].

It is worth emphasizing that the presentation (14) ideally suits for analysing resonant transitions between the coherent topological modes. And it is solely these modes that are 
the subject of the present paper. We shall not consider here other possible excitations that could be produced by nonresonant driving fields and studied by means of the known rescaling procedure. Nontopological nonresonant breathing-type oscillations have been considered by many authors in the early days of the BEC research (see reviews [1]-[5]). Therefore there is no reason of extending the paper by repeating similar results. This is why here we limit ourselves by treating only the resonant generation of topological modes, which have not been studied earlier.

Aiming at exciting particular modes, one should keep in mind, in addition to the resonance conditions (8), the symmetry properties of the corresponding wave functions, for which the modulating field has to be such that the transition amplitudes (12) be nonzero. However, even if all above mentioned conditions hold true, there exists a rather general situation when the generation of modes is impossible.

\section{No-Go Theorem for Mode Generation}

It may happen that the applied modulating field is not able to generate higher nonlinear modes, but can only lead to the oscillation of the initial wave function, without changing its shape. More precisely, let us start with a wave function $\varphi(\mathbf{r}, 0)$. After a modulating field $V(\mathbf{r}, t)$ begins acting on atoms, the function $\varphi(\mathbf{r}, 0)$ is transferred to a function $\varphi(\mathbf{r}, t)$. When the shape-conservation condition

$$
|\varphi(\mathbf{r}, t)|=|\varphi(\mathbf{r}-\mathbf{a}, 0)|
$$

holds true for time dependent $\mathbf{a}=\mathbf{a}(t)$, then the shape of the atomic cloud does not change in time, but the cloud oscillates as a whole, with its center of mass moving according to the dependence $\mathbf{a}(t)$. Hence, if we start with a mode $\varphi(\mathbf{r}, 0)$ it can never be transferred to another mode.

By definition, the initial function $\varphi(\mathbf{r}, 0)=\varphi_{0}(\mathbf{r})$ presents a nonlinear mode if it satisfies the stationary Gross-Pitaevskii equation

$$
\hat{H}\left[\varphi_{0}(\mathbf{r})\right] \varphi_{0}(\mathbf{r})=E_{0} \varphi_{0}(\mathbf{r})
$$

with the nonlinear Hamiltonian (2). We assume that this initial mode is real-valued, i.e.,

$$
\varphi(\mathbf{r}, 0)=\varphi_{0}(\mathbf{r})=\varphi_{0}^{*}(\mathbf{r}) .
$$

An example for this would be the ground-state of a Bose-Einstein condensate. In addition, we are focusing on trapped atoms, which implies that the confining potential $U(\mathbf{r})$ increases towards infinity for $r \equiv|\mathbf{r}| \rightarrow \infty$. Therefore, the trapping condition

$$
\lim _{\mathbf{r} \rightarrow \infty} \varphi(\mathbf{r}, t)=0
$$

is valid for all $t \geq 0$.

Theorem. Suppose that atoms in a trapping potential $U(\mathbf{r})$, being initially in a real mode $\varphi_{0}(\mathbf{r})$, are subject to the action of a modulating field $V(\mathbf{r}, t)$, so that conditions (17) to (19) are valid. Then the solution of the temporal Gross-Pitaevskii equation (11) 
preserves the shape of the initial mode, satisfying condition (16), if and only if the trapping potential is harmonic,

$$
U(\mathbf{r})=A_{0}+\mathbf{A}_{1} \cdot \mathbf{r}+\sum_{\alpha \beta} A_{\alpha \beta} r^{\alpha} r^{\beta}
$$

where $\alpha$ and $\beta$ are the Cartesian indices, while the modulating field is linear with respect to the spatial variables,

$$
V(\mathbf{r}, t)=B_{0}(t)+\mathbf{B}_{1}(t) \cdot \mathbf{r},
$$

$B_{0}(t)$ and $\mathbf{B}_{1}(t)$ being arbitrary functions of time. And the center-of-mass motion $\mathbf{a}=\mathbf{a}(t)$ is described by the equation

$$
m_{0} \frac{d^{2} a^{\alpha}}{d t^{2}}+\sum_{\beta}\left(A_{\alpha \beta}+A_{\beta \alpha}\right) a^{\beta}+B_{1}^{\alpha}(t)=0 .
$$

Proof. Assume that the shape-conservation condition (16) holds true, and let us show that then the trapping and modulating potentials are necessarily such as in Eqs. (20) and (21). For this purpose, it is useful to invoke the presentation

$$
\varphi(\mathbf{r}, t)=|\varphi(\mathbf{r}, t)| \exp \{i S(\mathbf{r}, t)\}
$$

where $S(\mathbf{r}, t)$ is a real action defining the velocity

$$
\mathbf{v}(\mathbf{r}, t) \equiv \frac{\hbar}{m_{0}} \vec{\nabla} S(\mathbf{r}, t)
$$

Substituting the presentation (23) into the GPE (11) yields [1] - [5] the continuity equation

$$
\frac{\partial}{\partial t}|\varphi|^{2}+\vec{\nabla}\left(|\varphi|^{2} \mathbf{v}\right)=0
$$

and the velocity equation

$$
m_{0} \frac{\partial \mathbf{v}}{\partial t}=-\vec{\nabla} U_{e f f}
$$

in which the effective potential

$$
U_{e f f}=U(\mathbf{r})+V(\mathbf{r}, t)+N A_{s}|\varphi|^{2}-\frac{\hbar^{2} \vec{\nabla}^{2}|\varphi|}{2 m_{0}|\varphi|}+\frac{m_{0} v^{2}}{2} .
$$

Because of the shape-conservation condition (16), one has

$$
\frac{\partial|\varphi|}{\partial t}=-\vec{\nabla}|\varphi| \cdot \frac{d \mathbf{a}}{d t} \text {. }
$$

Using this in the continuity equation (25) results in

$$
\vec{\nabla}\left[|\varphi|^{2}\left(\mathbf{v}-\frac{d \mathbf{a}}{d t}\right)\right]=0
$$


from where

$$
\mathbf{v}=\frac{d \mathbf{a}}{d t}+\frac{\mathbf{c}}{|\varphi|^{2}}, \quad \mathbf{c}=\mathbf{c}(t)
$$

If here the function $\mathbf{c}(t)$ is not zero, then from the trapping condition (19) it follows that $\mathbf{v} \rightarrow \infty$ as $r \rightarrow \infty$ hence, the action $S \rightarrow \infty$ as $r \rightarrow \infty$. But then the limit $r \rightarrow \infty$ of the function (23) is not defined. Therefore, $\mathbf{c}(t)=0$. Thus, the velocity (24) becomes

$$
\mathbf{v}=\frac{d \mathbf{a}}{d t}=\mathbf{v}(t)
$$

which is a function of time only. But then the velocity equation (26) tells us that $\vec{\nabla} U_{\text {eff }}$ is also a function of time only, hence the effective potential is linear in $\mathbf{r}$, having the form

$$
U_{e f f}=D_{0}(t)+\mathbf{D}_{1}(t) \cdot \mathbf{r}
$$

Taking into account that the initial mode is real-valued, as is conditioned by Eq. (18), and employing the shape-conservation condition (16), one gets

$$
|\varphi(\mathbf{r}, t)|=\varphi_{0}(\mathbf{r}-\mathbf{a}) .
$$

Then the effective potential (27) can be written as

$$
U_{e f f}=U(\mathbf{r})-U(\mathbf{r}-\mathbf{a})+V(\mathbf{r}, t)+E_{0}+\frac{m_{0} v^{2}}{2} .
$$

Since, according to Eq. (29), the effective potential is linear in $\mathbf{r}, E_{0}$ is a constant, and $v=v(t)$ is a function of time, then the sum of the first three terms in Eq. (31) should also be linear in $\mathbf{r}$, such that

$$
U(\mathbf{r})-U(\mathbf{r}-\mathbf{a})+V(\mathbf{r}, t)=U_{0}(t)+\mathbf{U}_{1}(t) \cdot \mathbf{r} .
$$

Thence the effective potential (31) takes the form

$$
U_{e f f}=E_{0}+\frac{m_{0} v^{2}}{2}+U_{0}(t)+\mathbf{U}_{1}(t) \cdot \mathbf{r}
$$

Comparing Eqs. (29) and (33), we have

$$
D_{0}(t)=E_{0}+\frac{m_{0} v^{2}}{2}+U_{0}(t), \quad \mathbf{D}_{1}(t)=\mathbf{U}_{1}(t) .
$$

Equality (32) can be satisfied only if the trapping and modulating potentials are given by Eqs. (20) and (21). Substituting the latter in Eq. (32), we find

$$
\begin{gathered}
U_{0}(t)=B_{0}(t)+\mathbf{a} \cdot \mathbf{A}-\sum_{\alpha \beta} A_{\alpha \beta} a^{\alpha} a^{\beta}, \\
U_{1}^{\alpha}(t)=B_{1}^{\alpha}(t)+\sum_{\beta}\left(A_{\alpha \beta}+A_{\beta \alpha}\right) a^{\beta} .
\end{gathered}
$$


Combining Eqs. (26), (28), (33), and (35), we obtain Eq. (22) for the center-of-mass motion.

Now let us show that Eqs. (20) and (21) are sufficient for the validity of the shapeconservation condition (16). Equations (25) and (26), with the effective potential (27) and with the initial conditions

$$
|\varphi(\mathbf{r}, 0)|=\varphi_{0}(\mathbf{r}), \quad \mathbf{v}(\mathbf{r}, 0)=0,
$$

are equivalent to the GPE (11) with the initial condition (18). The latter equation is a nonlinear Schrödinger equation, which, being complimented by the boundary condition (19), possesses a unique solution [44. Hence, Eqs. (25) and (26), with the same boundary condition (19), enjoy a unique solution. These equations, under conditions (20) and (21), do have a solution satisfying the shape-conservation condition (16), which, according to the aforesaid, is a unique solution. This concludes the proof.

One should not confuse the shape-conservation criterion derived here with the known result of the decoupled center-of-mass motion in a harmonic potential. The criterion of shape conservation shows when the wave function retains its shape under the action of an external alternating field and when the shape is not preserved. The center-of-mass motion is a trivial byproduct of our theorem. The principal point is the shape conservation. The derived criterion shows that the trapping potential may be harmonic, but, if the driving field is nonlinear, then, irrespectively of the center-of-mass motion, the shape of the wave function will not be conserved.

This theorem teaches us that if the trapping potential is harmonic, which is a standard situation, then the modulating field, being linear in $\mathbf{r}$, is not able to generate topological modes, even if its alternating temporal parts oscillate in an exact resonance with the corresponding transition frequencies. It is in full agreement with a nonlinear EhrenfestTheorem for the mean position and variance of the collective wavefunction [11]. To generate such modes, at least one of the conditions (20) or (21) has to be broken. But if a resonant alternating field is linear and the trapping potential is harmonic, then the real initial mode just moves in space, without changing its shape. We have checked this conclusion by numerically solving the GPE under conditions (20) and (21) and found a perfect agreement with the theorem.

\section{Coupling of Three Nonlinear Modes}

Several modes can be generated by applying a modulating field (6) containing several corresponding resonant terms. In general, two cases are admissible: when the excited modes are not coupled to each other and when they are coupled. In the former case, the overall dynamics consists of the motion of several pairs of modes, each pair being separated in its motion from other modes. The motion of such separate pairs of resonant modes has been studied in detail earlier [6, 8, 9, 10, 11, 14, 17, 21, 22. Therefore we now concentrate on the case of several coupled modes.

We consider the case of three coupled modes, whose transition frequencies (5) are enumerated as $\omega_{21}, \omega_{31}$, and $\omega_{32}$, keeping in mind that the related energy levels are such that $E_{1}<E_{2}<E_{3}$. To couple the modes, it is sufficient to have two modulating 
fields of the possible three that would be in resonance with the corresponding transition frequencies. In general, there can be three detunings $\Delta_{21}, \Delta_{31}$, and $\Delta_{32}$ satisfying the resonance conditions (8). For the transition amplitudes (12), we have $\beta_{12}, \beta_{13}$, and $\beta_{23}$. There are six amplitudes (11) for $\alpha_{i j}$ with $i \neq j$.

Realizing the coupling of three modes by two driving fields, we can create three types of resonant systems, which may be called, by analogy with the similar situations for resonant atoms, as cascade, $V$-type, and $\Lambda$-type systems [45]. In the cascade-type generation, the modes with the transition frequencies $\omega_{21}$ and $\omega_{32}$ are coupled. In the $V$-type case, the transition frequencies are $\omega_{21}$ and $\omega_{31}$. And for the $\Lambda$-type system, the transition frequencies are $\omega_{31}$ and $\omega_{32}$.

Substituting into the GPE (11) the presentation (14) and the corresponding driving fields (6), we employ the averaging technique [43. This procedure is absolutely the same as has been thoroughly described in [6, 8], so we do not repeat it here. The result is the system of equations for the coefficient functions

$$
\begin{aligned}
& i \frac{d c_{1}}{d t}=\left(\alpha_{12}\left|c_{2}\right|^{2}+\alpha_{13}\left|c_{3}\right|^{2}\right) c_{1}+F_{1}, \\
& i \frac{d c_{2}}{d t}=\left(\alpha_{21}\left|c_{1}\right|^{2}+\alpha_{23}\left|c_{3}\right|^{2}\right) c_{2}+F_{2} \\
& i \frac{d c_{3}}{d t}=\left(\alpha_{31}\left|c_{1}\right|^{2}+\alpha_{32}\left|c_{2}\right|^{2}\right) c_{3}+F_{3}
\end{aligned}
$$

where the terms $F_{i}$ depend on the type of the generation method. For the cascade generation, we have

$$
\begin{gathered}
F_{1}=\frac{1}{2} \beta_{12} c_{2} e^{i \Delta_{21} t}, \\
F_{2}=\frac{1}{2} \beta_{12}^{*} c_{1} e^{-i \Delta_{21} t}+\frac{1}{2} \beta_{23} c_{3} e^{i \Delta_{32} t}, \\
F_{3}=\frac{1}{2} \beta_{23}^{*} c_{2} e^{-i \Delta_{32} t} .
\end{gathered}
$$

If we set here $\alpha_{13}, \alpha_{31}, \alpha_{23}, \alpha_{32}$, and $\beta_{23}$ to zero, we return to the studied earlier two-mode case [6, 8]. In the case of the $V$-type coupling,

$$
\begin{gathered}
F_{1}=\frac{1}{2} \beta_{12} c_{2} e^{i \Delta_{21} t}+\frac{1}{2} \beta_{13} c_{3} e^{i \Delta_{31} t}, \\
F_{2}=\frac{1}{2} \beta_{12}^{*} c_{1} e^{-i \Delta_{21} t}, \quad F_{3}=\frac{1}{2} \beta_{13}^{*} c_{1} e^{-i \Delta_{31} t} .
\end{gathered}
$$

And for the $\Lambda$-type generation,

$$
\begin{gathered}
F_{1}=\frac{1}{2} \beta_{13} c_{3} e^{i \Delta_{31} t}, \quad F_{2}=\frac{1}{2} \beta_{23} c_{3} e^{i \Delta_{32} t}, \\
F_{3}=\frac{1}{2} \beta_{13}^{*} c_{1} e^{-i \Delta_{31} t}+\frac{1}{2} \beta_{23}^{*} c_{2} e^{-i \Delta_{32} t} .
\end{gathered}
$$

In addition, from the normalization of the function (14), we have

$$
\left|c_{1}\right|^{2}+\left|c_{2}\right|^{2}+\left|c_{3}\right|^{2}=1 \text {. }
$$


Each $c_{i}=c_{i}(t)$ defines the dynamics of the corresponding fractional mode population $\left|c_{i}\right|^{2}$.

It is important to stress that Eqs. (36) are obtained by employing the standard averaging method [43]), taking into account the existence of two time scales, slow and fast, related to the inequalities (13) and (15). In this averaging procedure, one substitutes expansion (14) into the GPE (11) and averages over time fastly oscillating functions. As a result of this, the so-called time-reversed or counter-rotating terms vanish, so that in the right-hand side of Eqs. (36) there are no terms like $c_{1}^{*} c_{2}^{2}$ or $c_{2}^{*} c_{3}^{2}$. It is well known that, even if such terms would be added, they do not produce a significant change in the solutions. Omitting these terms is essentially equivalent to the widely known rotating-wave approximation [45]. The derivation of equations for $c_{n}(t)$, with all related mathematical details has been thoroughly described in Refs. [6, 8, 22,

Though Eqs. (36) look differently for different types of mode generation related to distinct terms $F_{i}$, the mathematical structure of these equations is, actually, the same. We may notice the following symmetry properties. The $V$-type equations can be obtained from the cascade-type ones by interchanging the indices 1 and 2 and by replacing $\beta_{21}$ by $\beta_{12}^{*}$. Similarly, the $\Lambda$-type equations can be derived from the cascade-type equations by interchanging the indices 2 and 3 , with the replacement $\beta_{32} \rightarrow \beta_{23}^{*}$. The relation $\Delta_{i j}=-\Delta_{j i}$ has to be taken into account. Because of this symmetry, it is sufficient to consider just one type of Eqs. (36), for instance, that corresponding to the cascade generation.

The functions $c_{i}(t)$ are complex-valued. Hence, Eqs. (36) present a system of six differential equations. However, it is possible to show that they define a four-dimensional dynamical system. To prove this, we involve the notation

$$
c_{j}=\left|c_{j}\right| \exp \left(i \pi_{j}\right)
$$

where $\pi_{j}=\pi_{j}(t)$ is a real-valued phase. Also, we write

$$
\beta_{i j}=b_{i j} \exp \left(i \gamma_{i j}\right), \quad b_{i j} \equiv\left|\beta_{i j}\right|
$$

Introduce the population differences

$$
s \equiv\left|c_{2}\right|^{2}-\left|c_{1}\right|^{2}, \quad p \equiv\left|c_{3}\right|^{2}-\left|c_{2}\right|^{2}
$$

and the relative phases

$$
x \equiv \pi_{2}-\pi_{1}+\gamma_{12}+\Delta_{21} t, \quad y \equiv \pi_{3}-\pi_{2}+\gamma_{23}+\Delta_{32} t
$$

The fractional mode populations can be expressed through the variables (43) as

$$
\left|c_{1}\right|^{2}=\frac{1}{3}(1-2 s-p), \quad\left|c_{2}\right|^{2}=\frac{1}{3}(1+s-p) \quad\left|c_{3}\right|^{2}=\frac{1}{3}(1+s+2 p) .
$$

Then Eqs. (36) for the cascade generation can be reduced to the system of four equations

$$
\frac{d s}{d t}=\frac{1}{3} \sqrt{1+s-p}\left(b_{23} \sqrt{1+s+2 p} \sin y-2 b_{12} \sqrt{1-2 s-p} \sin x\right),
$$




$$
\begin{gathered}
\frac{d p}{d t}=\frac{1}{3} \sqrt{1+s-p}\left(b_{12} \sqrt{1-2 s-p} \sin x-2 b_{23} \sqrt{1+s+2 p} \sin y\right), \\
\frac{d x}{d t}=\alpha_{1} s+\delta_{1} p+\frac{3 b_{12} s \cos x}{2 \sqrt{(1+s-p)(1-2 s-p)}}-\frac{1}{2} b_{23} \sqrt{\frac{1+s+2 p}{1+s-p}} \cos y+\delta_{2}, \\
\frac{d y}{d t}=\alpha_{2} p+\delta_{3} s+\frac{3 b_{23} p \cos y}{2 \sqrt{(1+s-p)(1+s+2 p)}}+\frac{1}{2} b_{12} \sqrt{\frac{1-2 s-p}{1+s-p}} \cos x+\delta_{4},
\end{gathered}
$$

in which

$$
\begin{array}{cc}
\alpha_{1} \equiv \frac{1}{3}\left(\alpha_{12}+\alpha_{13}+2 \alpha_{21}-\alpha_{23}\right), & \alpha_{2} \equiv \frac{1}{3}\left(\alpha_{32}+\alpha_{31}+2 \alpha_{23}-\alpha_{21}\right), \\
\delta_{1} \equiv \frac{1}{3}\left(\alpha_{21}-\alpha_{12}+2 \alpha_{13}-2 \alpha_{23}\right), & \delta_{2} \equiv \Delta_{21}+\frac{1}{3}\left(\alpha_{12}-\alpha_{21}+\alpha_{13}-\alpha_{23}\right), \\
\delta_{3} \equiv \frac{1}{3}\left(\alpha_{23}-\alpha_{32}+2 \alpha_{31}-2 \alpha_{21}\right), & \delta_{4} \equiv \Delta_{32}+\frac{1}{3}\left(\alpha_{23}-\alpha_{32}+\alpha_{21}-\alpha_{31}\right) .
\end{array}
$$

Thus, the set of six equations (36) is really equivalent to a four-dimensional dynamical system (45).

We have numerically investigated the behaviour of solutions to Eqs. (45) for various parameters $\alpha_{i}, b_{i j}$, and $\delta_{i}$. The latter parameter, playing the role of an effective detuning, was assumed to be small, $\delta_{i} \ll 1$. Different initial conditions have been considered in the range $-1 \leq s_{0} \leq 1,-1 \leq p_{0} \leq 1,0 \leq x_{0} \leq 2 \pi, 0 \leq y_{0} \leq 2 \pi$. For small $b_{i j} \ll \alpha_{i}$, the solutions for the population differences $s$ and $p$ demonstrate a kind of nonlinear Rabi oscillations in the mode locked regime, when $s(t)$ and $p(t)$ do not cross the zero line, being always either above or below it, depending on initial conditions. This mode locked regime is the same as in the case of two modes, studied earlier [8, 14, 17, 21, 22. The difference with the two-mode case is that the Rabi-type oscillations look slightly more complicated, being quasiperiodic but not periodic. Increasing $b_{i j}$ results in the increase of the oscillation amplitudes, and, after a critical value of $b_{i j}$, the mode unlocked regime arises, when either $s(t)$, or $p(t)$, or both of them, oscillate in the whole interval $[-1,1]$. To illustrate these two regimes, we present numerical calculations accomplished for equal parameters $\alpha_{i j}=\alpha$ with the initial conditions $s_{0}=-1, p_{0}=0, x_{0}=y_{0}=0$. Figure [1] shows the mode locked regime, when $s(t)<0$ for all $t \geq 0$, and $p(t)$ is also negative for almost all $t$. Figure 2 demonstrates the mode unlocked regime, when $s(t) \in[-1,1]$, while $p(t)$ is yet almost always negative. Increasing further $b_{i j}$ leads to the situation, when $p(t)$ starts also oscillating in the interval $[-1,1]$. However, at large $b_{i j}$, the temporal behaviour of solutions becomes quite unstable resembling chaotic motion. To better understand what happens, it is necessary to study the phase portrait of the dynamical system, that is, one has to find the fixed points and to analyze their stability. However, before turning to this task, we will compare the results of the averaging method described above with those of a direct numerical simulation of the GPE for several special cases.

\section{Direct numerical simulation of time evolution}

Although the averaging technique is a well grounded mathematical method [43, 46], it is interesting to show that the main effects, described above, also appear in a direct 
numerical simulation of the time evolution described by Eq. (11). One important reason is that the results of the averaging procedure are basically independent of the details of the trapping potential, while a direct numerical simulation is affected by it. We consider two cases: in an anharmonic potential the conditions for the validity of Eq. (36) can well be met and reasonable agreement can be expected. On the other hand, in a harmonic trap the averaging method may break down. We will demonstrate both situations using the numerical methods and parameter settings which are described in the Appendix.

\subsection{Anharmonic trap with linear driving field}

The case of an anharmonic trapping potential is most suited to demonstrate the benefits of the few-mode averaging method. We considered a BEC moving in a potential of the form $U_{0} z^{4}$ with $U_{0}=10^{-32} \mathrm{~J} / \mu \mathrm{m}^{4}$ and a linear driving potential of the form $V_{1}=\beta_{1} z$ (in the notation of Eq. (6) $)$ which was tuned to resonance with the $\varphi_{0} \rightarrow \varphi_{1}$ transition so that $\omega_{1}=\left(E_{1}-E_{0}\right) / \hbar \approx 610 / \mathrm{s}$. In absence of the driving potential the BEC can occupy several stationary nonlinear coherent modes, three of which are displayed in Fig. 3. The respective parameters appearing in Eq. (6) are found to be $\alpha_{12}=121.7 \mathrm{~s}^{-1}, \alpha_{13}=46.3 \mathrm{~s}^{-1}$, $\alpha_{21}=144.2 s^{-1}, \alpha_{23}=88.9 s^{-1}, \alpha_{31}=91.8 s^{-1}$, and $\alpha_{32}=111.9 s^{-1}$.

As predicted by the averaging method our simulation showed a critical behaviour: For $\beta_{1}$ below a certain value $\beta_{c} \approx 7.3 \times 10^{-33} \mathrm{~J} / \mu \mathrm{m}$ the population transferred to the first excited state is bounded to be smaller than about 0.5. An example for this behaviour can be seen in Fig. 4 a) which corresponds to a slightly subcritical value of $\beta_{1}=6.9 \times 10^{-33}$ $\mathrm{J} / \mu \mathrm{m}$. For $\beta_{1}>\beta_{c}$ this upper bound suddenly disappears and the population of the first excited states can take almost all values between 0 and 1 . This can be seen in Fig. 5 a) which displays the time evolution for a slightly supercritical driving field with $\beta_{1}=7.7 \times 10^{-33} \mathrm{~J} / \mu \mathrm{m}$.

The corresponding predictions of the averaging technique can be seen in Figs $4 \mathrm{~b}$ ) and $5 \mathrm{~b})$, respectively. Since the period and amplitude of the populations $\left|c_{i}\right|^{2}$ are strongly varying around the critical value of $\beta_{1}$, we have chosen the values $\beta_{1}=1.0 \times 10^{-32} \mathrm{~J} / \mu \mathrm{m}$ for $4 \mathrm{~b}$ ) and $\beta_{1}=1.02 \times 10^{-32} \mathrm{~J} / \mu \mathrm{m}$ for $5 \mathrm{~b}$ ), which are close to the critical value as predicted by the averaging method. The found critical values of $\beta_{c}$ are in reasonable agreement, the difference being of about $25 \%$, which is, actually, the accuracy of the averaging technique for the given set of parameters.

\subsection{Harmonic trap with anharmonic driving field}

This case serves as an example of how the breakdown of the conditions (13) and (15) results in wrong predictions of the mode expansion method. The case of a harmonic trap is a very special case with this respect: if, in absence of interaction, the transition between two neighbouring modes is resonantly driven, then this is also the case for a transition between any other neighbouring modes. It therefore is never possible to consider only a small number of states because other states are quickly populated as well. Only if one is sufficiently far away from resonance a few-mode model can be expected to work well, but then the transition rate between those modes is also low. These conclusions remain qualitatively also valid in the presence of interaction. 
To demonstrate the breakdown of the mode expansion method we consider a harmonic potential of the form $U_{z}(z)=m_{0} \omega_{z}^{2} z^{2} / 2$ with $\omega_{z}=600 / \mathrm{s}$, and a screened cubic driving field which, in the notation of Eq. ([6)), is given by $V_{i}(z)=\beta_{i} z^{3} \exp \left(-z^{2} / w^{2}\right), i=1,2$, with $w=4.9 \mu \mathrm{m}$. We introduced the exponential screening since a purely cubic driving field plus a harmonic potential is unbounded from below. A linear driving field cannot be used because, according to the theorem given above, only leads to an oscillation of the BEC without changing its shape. The two driving frequencies are chosen to be at resonance with the $\varphi_{0} \rightarrow \varphi_{1}$ transition, $\omega_{1}=\left(E_{1}-E_{0}\right) / \hbar \approx 536 / \mathrm{s}$, and the $\varphi_{1} \rightarrow \varphi_{2}$ transition, $\omega_{2}=\left(E_{2}-E_{1}\right) / \hbar \approx 568 / \mathrm{s}$.

The relative strength of the two driving fields was chosen to achieve $\left(\varphi_{1}, V_{1} \varphi_{0}\right)=$ $\left(\varphi_{2}, V_{2} \varphi_{1}\right)$ by setting the appropriate values for $\beta_{1}$ and $\beta_{2}$. The results are shown in Fig. 6. For weak driving fields, $\beta_{1}=0.7869 \times 10^{-33} \mathrm{~J} / \mu \mathrm{m}^{3}$ and $\beta_{2}=0.344 \times 10^{-33}$ $\mathrm{J} / \mu \mathrm{m}^{3}$, one achieves reasonable agreement with the predictions of the averaging procedure, see Fig. [6 $)$. For a strong driving force with $\beta_{1}=1.96734 \times 10^{-33} \mathrm{~J} / \mu \mathrm{m}^{3}$ and $\beta_{2}=$ $0.859975 \times 10^{-33} \mathrm{~J} / \mu \mathrm{m}^{3}$ the excitation of higher modes becomes significant, which is not surprising because the driving field provides more energy which can also excite higher levels. This behaviour can be seen in Fig. [6b) which also indicates that the time evolution deviates strongly from the predictions of the averaging method.

\section{Stationary Solutions and Stability Analysis}

To find out the stationary solutions for the dynamics of the three-mode case and to analyse the stability of these solutions, it is convenient to work with the variables

$$
f_{j} \equiv\left|c_{j}\right| \quad(j=1,2,3)
$$

and the relative phases (44). The fractional mode populations are expressed through the amplitudes (46) as $n_{j}=f_{j}^{2}$.

Using the variables $f_{j}, x$, and $y$ one can rewrite Eqs. (36) for the cascade generation in the form

$$
\begin{gathered}
\frac{d f_{1}}{d t}=\frac{1}{2} b_{12} f_{2} \sin x \\
\frac{d f_{2}}{d t}=-\frac{1}{2} b_{12} f_{1} \sin x+\frac{1}{2} b_{23} f_{3} \sin y \\
\frac{d f_{3}}{d t}=-\frac{1}{2} b_{23} f_{2} \sin y \\
\frac{d x}{d t}=\Delta_{21}-\alpha_{21} f_{1}^{2}+\alpha_{12} f_{2}^{2}+\left(\alpha_{13}-\alpha_{23}\right) f_{3}^{2}+b_{12} \frac{f_{2}^{2}-f_{1}^{2}}{2 f_{1} f_{2}} \cos x-b_{23} \frac{f_{3}}{2 f_{2}} \cos y \\
\frac{d y}{d t}=\Delta_{32}+\left(\alpha_{21}-\alpha_{31}\right) f_{1}^{2}-\alpha_{32} f_{2}^{2}+\alpha_{23} f_{3}^{2}+b_{12} \frac{f_{1}}{2 f_{2}} \cos x+b_{23} \frac{f_{3}^{2}-f_{2}^{2}}{2 f_{2} f_{3}} \cos y .
\end{gathered}
$$

There are here yet too many parameters, because of which the analysis of Eqs. (477) is yet too complicated. To simplify the consideration, we may involve a realistic approximation, 
when there is no detuning, the amplitudes $b_{i j}$, due to the applied alternating fields, are taken to be the same, and the parameters $\alpha_{i j}$ are close to each other. That is, we set

$$
\alpha \equiv \alpha_{i j}, \quad b \equiv \frac{b_{i j}}{\alpha}, \quad \Delta_{i j}=0
$$

It is also convenient to measure time in units of $\alpha^{-1}$. To return back to time units, one should replace $t$ by $\alpha t$. Then Eqs. (47) reduce to

$$
\begin{gathered}
\frac{d f_{1}}{d t}=\frac{b}{2} f_{2} \sin x, \\
\frac{d f_{2}}{d t}=-\frac{b}{2} f_{1} \sin x+\frac{b}{2} f_{3} \sin y, \\
\frac{d f_{3}}{d t}=-\frac{b}{2} f_{2} \sin y, \\
\frac{d x}{d t}=f_{2}^{2}-f_{1}^{2}+b \frac{f_{2}^{2}-f_{1}^{2}}{2 f_{1} f_{2}} \cos x-b \frac{f_{3}}{2 f_{2}} \cos y, \\
\frac{d y}{d t}=f_{3}^{2}-f_{2}^{2}+b \frac{f_{1}}{2 f_{2}} \cos x+b \frac{f_{3}^{2}-f_{2}^{2}}{2 f_{2} f_{3}} \cos y .
\end{gathered}
$$

In addition, because of Eqs. (40) and (46), there is the relation

$$
f_{1}^{2}+f_{2}^{2}+f_{3}^{2}=1
$$

This relation is automatically supported by Eqs. (49) as well as by Eqs. (47), provided it is valid for the initial $f_{i}(0)$. Equations (49) are much easier to analyse than Eqs. (47). At the same time, the mathematical structure of these equations is similar, so the behaviour of their solutions should be close to each other.

In Eqs. (49), there is the sole dimensionless parameter b, defined in Eq. (48). Since the parameter $\alpha$ can be positive as well as negative, then $b$ can, generally, be of both signs too. But Eqs. (49) enjoy a nice symmetry property, being invariant under the change

$$
b \rightarrow-b, \quad x \rightarrow x \pm \pi, \quad y \rightarrow y \pm \pi .
$$

Therefore, in what follows, it is sufficient to consider only the case of positive $b \geq 0$.

There exists a special solution of Eqs. (49), for which

$$
f_{1}=f_{3}, \quad x=-y
$$

for all times $t \geq 0$. Then the problem reduces to a kind of a two-mode case described by the equations

$$
\begin{gathered}
\frac{d f_{1}}{d t}=\frac{b}{2} f_{2} \sin x \\
\frac{d f_{2}}{d t}=-b f_{1} \sin x \\
\frac{d x}{d t}=f_{2}^{2}-f_{1}^{2}+b \frac{2 f_{2}^{2}-1}{2 f_{1} f_{2}} \cos x
\end{gathered}
$$


This reduction, however, becomes possible as a result of the approximation when all interaction amplitudes $\alpha_{i j}=\alpha$ are assumed to be equal. More precisely, the minimal requirements necessary for the existence of solution (52) are

$$
\alpha_{12}=\alpha_{32}, \quad \alpha_{13}=\alpha_{31}, \quad b_{12}=b_{23}, \quad \Delta_{21}=\Delta_{32} .
$$

It is feasible, in principle, to choose such modulating fields and a trapping potential that Eqs. (53) be valid. In addition, the initial conditions are to be such that $f_{1}(0)=f_{3}(0)$.

The stationary solutions of Eqs. (49) are obtained by equating to zero their righthand sides, keeping in mind that $b \neq 0$ and $f_{2}$ is not identically zero. The corresponding fixed-point equations for the phase differences are

$$
\sin x=\sin y=0, \quad \cos x= \pm 1, \quad \cos y= \pm 1 .
$$

The equations for the amplitudes are rather cumbersome, and we shall not write them down. We shall solve these equations numerically, calculating the fixed-point amplitudes $f_{i}^{*}=f_{i}^{*}(b)$ as functions of the pumping parameter $b$ and then finding the related stationary solutions

$$
n_{i}^{*}(b) \equiv\left|f_{i}^{*}(b)\right|^{2}
$$

for the fractional mode populations. Simultaneously, we accomplish the stability analysis by calculating the Jacobian matrix for Eqs. (49), evaluated at the related fixed points. The eigenvalues of this matrix define the characteristic exponents characterizing the type of stability [47].

The first stationary solution is given by $x^{*}=y^{*}=0$ and $n_{1}^{*}=n_{3}^{*}$. The related mode populations (55) are shown in Fig. 7 This fixed point is neutrally stable, being a center with the characteristic exponents $\Lambda_{1}=0$ and imaginary $\Lambda_{2}=\Lambda_{3}^{*}, \Lambda_{4}=\Lambda_{5}^{*}$. When $b$ varies in the interval $0.1 \leq b \leq 1$, the absolute values of $\Lambda_{2,3}$ and $\Lambda_{4,5}$ change in the range $0.192 \leq\left|\Lambda_{2,3}\right| \leq 0.865$ and $0.351 \leq\left|\Lambda_{4,5}\right| \leq 1.755$.

The second fixed point is defined by $x^{*}=y^{*}=\pi$ and the branch I in Fig. 8 a) for $n_{1}^{*}=n_{3}^{*}$ and in Fig. 8b) for $n_{2}^{*}$. This fixed point is also a center with the characteristic exponents $\Lambda_{1}=0$ and imaginary $\Lambda_{2}=\Lambda_{3}^{*}, \Lambda_{4}=\Lambda_{5}^{*}$. For $b \in[0,1]$, one has $\left|\Lambda_{2,3}\right| \sim$ $\left|\Lambda_{4,5}\right| \sim 1$.

The third fixed point corresponds to $x^{*}=y^{*}=\pi$ and the branch II in Figs. 8 a) and 8b) for $n_{1}^{*}=n_{3}^{*}$ and $n_{2}^{*}$, respectively. The characteristic exponents are $\Lambda_{1}=0$ and real $\Lambda_{2}=-\Lambda_{3}, \Lambda_{4}=-\Lambda_{5}$, which shows that this point is unstable. The absolute values are $\left|\Lambda_{2,3}\right| \sim\left|\Lambda_{4,5}\right| \sim 0.1$. The solution exists for $0 \leq b \leq b_{0}^{*}$, with $b_{0}^{*}=0.198431$.

The fourth fixed point is given by $x^{*}=y^{*}=\pi$ and the branch III in Figs. 8 8 a) and [8]) for $n_{1}^{*}=n_{3}^{*}$ and $n_{2}^{*}$. The characteristic exponents are $\Lambda_{1}=0$, imaginary $\Lambda_{2}=\Lambda_{3}^{*}$, and real $\Lambda_{4}=-\Lambda_{5}$, with $\left|\Lambda_{2,3}\right| \sim\left|\Lambda_{4,5}\right| \sim 0.1$. This solution is unstable and exists under $0 \leq b \leq b_{0}^{*}$, with $b_{0}^{*}=0.198431$.

The fifth fixed point is described by $x^{*}=y^{*}=\pi$ and the branch I in Fig. 9 for $n_{1}^{*}(b)$, $n_{2}(b)$, and $n_{3}^{*}(b)$, respectively. The characteristic exponents are $\Lambda_{1}=0$ and imaginary $\Lambda_{2}=\Lambda_{3}^{*}, \Lambda_{4}=\Lambda_{5}^{*}$, which shows that this point is a center. The solution exists for $b$ in the range $0 \leq b \leq b_{c}^{*}$, with $b_{c}^{*}=0.639448$, where $\left|\Lambda_{2,3}\right| \sim 1$ and $\left|\Lambda_{4,5}\right| \leq 0.967$.

The sixth fixed point corresponds to $x^{*}=y^{*}=\pi$ and the branch II in Fig. 9 for $n_{1}^{*}(b)$, $n_{2}^{*}(b)$, and $n_{3}^{*}(b)$. The related characteristic exponents are $\Lambda_{1}=0$, imaginary $\Lambda_{2}=\Lambda_{3}^{*}$, and 
real $\Lambda_{4}=-\Lambda_{5}$. This tells that this point is unstable. The solution exists for $0 \leq b \leq b_{c}^{*}$, with $b_{c}^{*}=0.639448$, where $\left|\Lambda_{2,3}\right| \leq 0.896$ and $\left|\Lambda_{4,5}\right| \leq 0.457$.

The stationary solutions for the case $n_{1}^{*} \neq n_{3}^{*}$ are, actually, invariant under the interchange of the indices 1 and 3 . For concreteness, we accept that $n_{1}^{*}>n_{3}^{*}$, as is shown in Fig. 9. Formally, there is also the seventh fixed point, for which $x^{*}=y^{*}=2 \pi$ and $n_{1}^{*}=n_{3}^{*}$. But this is just the first point shifted in $x^{*}$ and $y^{*}$ by $2 \pi$.

Selecting from all available fixed points only those that corresponds to stable stationary solutions, we have three such cases: the point $x^{*}=y^{*}=0, n_{1}^{*}=n_{3}^{*}$, depicted in Fig. [7] the point $x^{*}=y^{*}=\pi, n_{1}^{*}=n_{3}^{*}$, shown in Fig. 8 as the branch I; and the point $x^{*}=y^{*}=\pi$, $n_{1}^{*}>n_{3}^{*}$, presented as the branch I in Fig. 9.

Recall that these three stable fixed points exist under the validity of conditions (153). In reality, it can be quite difficult to satisfy these conditions exactly. But if these conditions are not valid, then the sole stable point that remains is the point $x^{*}=y^{*}=\pi, n_{1}^{*}>n_{3}^{*}$, which is shown as the branch I in Fig. 9. This stationary solution exists only for $b \leq b_{c}^{*}$. For larger pumping parameters $b>b_{c}^{*}$, there are no stable (or neutrally stable) fixed points. Hence, the motion for such large $b$ will be chaotic.

\section{Harmonic Generation and Parametric Conversion}

Employing the presentation (14) in the GPE (11), and involving the averaging technique [43, we come to the equations for $c_{n}(t)$, like Eqs. (36). This procedure defines an initial approximation for $c_{n}(t)$, which can be called the guiding centers and labelled as $c_{n}^{(0)}(t)$. It is possible to obtain corrections to the guiding centers by using the following steps of the averaging technique [43, 46]. Then we can find the higher approximations $c_{n}^{(k)}(t)$ describing the fractional mode populations $\left|c_{n}^{(k)}(t)\right|^{2}$. To obtain the higher approximations for $c_{n}(t)$, we may proceed as follows.

Let us present the solution to the temporal equation (11) as

$$
\varphi(\mathbf{r}, t)=\sum_{n} c_{n}(t) \varphi_{n}(\mathbf{r}, t)
$$

where again $c_{n}(t)$ is a slow function of time, compared to the fast function $\varphi_{n}(\mathbf{r}, t)$. For any fast function of time $F(t)$, we define the averaging

$$
<F>\equiv \lim _{\tau \rightarrow \infty} \frac{1}{\tau} \int_{0}^{\tau} F(t) d t
$$

over fast oscillations. In particular,

$$
<\exp \left\{i\left(\omega_{1}-\omega_{2}\right) t\right\}>=\Delta\left(\omega_{1}-\omega_{2}\right) \equiv \begin{cases}1, & \omega_{1}=\omega_{2} \\ 0, & \omega_{1} \neq \omega_{2}\end{cases}
$$

The functions $\varphi_{n}(\mathbf{r}, t)$ can be taken such that

$$
<\int \varphi_{m}^{*}(\mathbf{r}, t) \varphi_{n}(\mathbf{r}, t) d \mathbf{r}>=\delta_{m n} .
$$

Then the amplitudes $c_{n}(t)$ in Eq. (56) can be obtained as

$$
c_{n}(t)=<\int \varphi_{n}^{*}(\mathbf{r}, t) \varphi(\mathbf{r}, t) d \mathbf{r}>
$$


and the normalization

$$
\sum_{n}\left|c_{n}(t)\right|^{2}=1
$$

is valid. For instance, taking $\varphi_{n}(\mathbf{r}, t)$ in the form

$$
\varphi_{n}^{(0)}(\mathbf{r}, t)=\varphi_{n}(\mathbf{r}) \exp \left(-\frac{i}{\hbar} E_{n} t\right)
$$

we come back to Eq. (14), with all Eqs. (158) to (60) being evidently satisfied.

Equation (59) can be employed as a relation for an iterative procedure defined by the rule

$$
c_{n}^{(k+1)}(t)=<\int \varphi_{n}^{(k) *}(\mathbf{r}, t) \varphi^{(k)}(\mathbf{r}, t) d \mathbf{r}>,
$$

where

$$
\varphi^{(k)}(\mathbf{r}, t) \equiv \sum_{n} c_{n}^{(k)}(t) \varphi_{n}^{(k)}(\mathbf{r}, t)
$$

Starting from the guiding centers $c_{n}^{(0)}(t)$ and the form (61), we get

$$
c_{n}^{11}(t)=c_{n}^{(0)}(t)
$$

which follows from the rule (62).

To derive the second-order approximation for $c_{n}(t)$, we write

$$
\varphi \frac{1}{n}(\mathbf{r}, t)=\left[\varphi_{n}(\mathbf{r})+\chi_{n}(\mathbf{r}, t)\right] \exp \left(-\frac{i}{\hbar} E_{n} t\right)
$$

Here $\varphi_{n}(\mathbf{r})$ is a stationary topological mode given by the eigenproblem (4), and $\chi_{n}(\mathbf{r}, t)$ has to be found by substituting Eq. (65) into the GPE (11). In the case of modulating field (9), the correcting term $\chi_{n}(\mathbf{r}, t)$ can be written as

$$
\chi_{n}(\mathbf{r}, t)=\sum_{j}\left[u_{n j}(\mathbf{r}) e^{-i \omega_{j} t}+v_{n j}^{*}(\mathbf{r}) e^{i \omega_{j} t}\right],
$$

where $j=1,2, \ldots$, and all $\omega_{j}>0$ can be ordered so that $0 \leq \omega_{1} \leq \omega_{2} \leq \ldots$ The functions $u_{n j}(\mathbf{r})$ and $v_{n j}(\mathbf{r})$ satisfy the equations

$$
\begin{gathered}
\left(\hat{H}\left[\varphi_{n}\right]-E_{n}+N A_{s}\left|\varphi_{n}\right|^{2}-\hbar \omega_{j}\right) u_{n j}+N A_{s} \varphi_{n}^{2} v_{n j}=-\frac{1}{2} \varphi_{n} B_{j}^{*}, \\
\left(\hat{H}\left[\varphi_{n}\right]-E_{n}+N A_{s}\left|\varphi_{n}\right|^{2}+\hbar \omega_{j}\right) v_{n j}+N A_{s}\left(\varphi_{n}^{*}\right)^{2} u_{n j}=-\frac{1}{2} \varphi_{n}^{*} B_{j},
\end{gathered}
$$

where $\varphi_{n}=\varphi_{n}(\mathbf{r})$. Using the first-order form (65) in Eqs. (63) and (62), we obtain the second-order approximation

$$
\begin{gathered}
c_{n}^{[2]}(t)=c_{n}^{(0)}(t)+\sum_{m} c_{m}^{(0)}(t)\left\{\sum_{i}\left[\left(\varphi_{n}, u_{m i}\right)+\left(v_{n i}^{*}, \varphi_{m}\right)\right] \Delta\left(\omega_{i}-\omega_{n m}\right)+\right. \\
\sum_{i}\left[\left(\varphi_{n}, v_{m i}^{*}\right)+\left(u_{n i}, \varphi_{m}\right)\right] \Delta\left(\omega_{i}+\omega_{n m}\right)+
\end{gathered}
$$




$$
\begin{aligned}
& +\sum_{i j}\left[\left(u_{n i}, u_{m j}\right) \Delta\left(\omega_{i}-\omega_{j}+\omega_{n m}\right)+\left(u_{n i}, v_{m j}^{*}\right) \Delta\left(\omega_{i}+\omega_{j}+\omega_{n m}\right)+\right. \\
& \left.\left.+\left(v_{n i}^{*}, u_{m j}\right) \Delta\left(\omega_{i}+\omega_{j}-\omega_{n m}\right)+\left(v_{n i}^{*}, v_{m j}^{*}\right) \Delta\left(\omega_{i}-\omega_{j}-\omega_{n m}\right)\right]\right\}
\end{aligned}
$$

in which $(u, v)$ denotes the corresponding scalar product. This formula is valid for an arbitrary number of alternating resonant fields. In the case of just one resonant field, there should be no summation over the indices $i$ and $j$ in Eq. (68). Formula (68) shows what are the conditions on the alternating fields, which induce resonant transitions between topological modes. These conditions depend on the number of the modulating fields involved.

Consider the case of two resonantly coupled topological modes, when

$$
c_{n}^{(0)}(t)=0 \quad(n \neq 1,2)
$$

and is nonzero only for $n=1,2$, as follows from the averaging technique for the guiding centers [6, 8]. And suppose that there is the sole alternating field with a frequency $\omega_{1} \equiv \omega$. Then the nontrivial population amplitudes are

$$
\begin{aligned}
c_{1}^{[2]}(t) & =c_{1}^{(0)}(t)+c_{2}^{(0)}(t)\left[\left(\varphi_{1}, v_{2}^{*}\right)+\left(u_{1}, \varphi_{2}\right)\right] \Delta\left(\omega-\omega_{21}\right)+c_{2}^{(0)}(t)\left(u_{1}, v_{2}^{*}\right) \Delta\left(2 \omega-\omega_{21}\right), \\
c_{2}^{[2]}(t) & =c_{2}^{(0)}(t)+c_{1}^{(0)}(t)\left[\left(\varphi_{2}, u_{1}\right)+\left(v_{2}^{*}, \varphi_{1}\right)\right] \Delta\left(\omega-\omega_{21}\right)+c_{1}^{(0)}(t)\left(v_{2}^{*}, u_{1}\right) \Delta\left(2 \omega-\omega_{21}\right),
\end{aligned}
$$

which results from Eq. (68), taking into account that $\omega_{12}=-\omega_{21}$.

If there are two alternating fields, so that $j=1,2$ in Eq. (68), then for the nonzero population amplitudes, one has

$$
\begin{gathered}
c_{1}^{\sqrt[2]{2}}=c_{1}^{(0)}(t)+c_{2}^{(0)}\left\{\left[\left(\varphi_{1}, v_{21}^{*}\right)+\left(u_{11}, \varphi_{2}\right)\right] \Delta\left(\omega_{1}-\omega_{21}\right)+\right. \\
+\left[\left(\varphi_{1}, v_{22}^{*}\right)+\left(u_{12}, \varphi_{2}\right)\right] \Delta\left(\omega_{2}-\omega_{21}\right)+\left(u_{11}, v_{21}^{*}\right) \Delta\left(2 \omega_{1}-\omega_{21}\right)+\left(u_{12}, v_{22}^{*}\right) \Delta\left(2 \omega_{2}-\omega_{21}\right)+ \\
\left.+\left[\left(u_{11}, v_{22}^{*}\right)+\left(u_{12}, v_{21}^{*}\right)\right] \Delta\left(\omega_{1}+\omega_{2}-\omega_{21}\right)+\left[\left(u_{12}, u_{21}\right)+\left(v_{22}, v_{11}\right)\right] \Delta\left(\omega_{2}-\omega_{1}-\omega_{21}\right)\right\} \\
c_{2}^{[2]}=c_{2}^{(0)}(t)+c_{1}^{(0)}\left\{\left[\left(\varphi_{2}, u_{11}\right)+\left(v_{21}^{*}, \varphi_{1}\right)\right] \Delta\left(\omega_{1}-\omega_{21}\right)+\right. \\
+\left[\left(\varphi_{2}, u_{12}\right)+\left(v_{22}^{*}, \varphi_{1}\right)\right] \Delta\left(\omega_{2}-\omega_{21}\right)+\left(v_{21}^{*}, u_{11}\right) \Delta\left(2 \omega_{1}-\omega_{21}\right)+\left(v_{22}^{*}, u_{12}\right) \Delta\left(2 \omega_{2}-\omega_{21}\right)+ \\
\left.+\left[\left(v_{21}^{*}, u_{12}\right)+\left(v_{22}^{*}, u_{11}\right)\right] \Delta\left(\omega_{1}+\omega_{2}-\omega_{21}\right)+\left[\left(u_{21}, u_{12}\right)+\left(v_{11}, v_{22}\right)\right] \Delta\left(\omega_{2}-\omega_{1}-\omega_{21}\right)\right\} .
\end{gathered}
$$

Expressions (70) show that one alternating field, with a frequency $\omega$, can induce transitions between two topological modes, provided that one of the following equations is valid:

$$
\omega=\omega_{21}, \quad 2 \omega=\omega_{21} .
$$

And Eqs. (71) demonstrate that two alternating fields, with frequencies $\omega_{1}$ and $\omega_{2}$, can realize intermode transitions under one of the resonance conditions:

$$
\begin{aligned}
\omega_{1} & =\omega_{21}, & \omega_{2}=\omega_{21}, \\
2 \omega_{1} & =\omega_{21}, & 2 \omega_{2}=\omega_{21},
\end{aligned}
$$




$$
\omega_{1}+\omega_{2}=\omega_{21}, \quad \omega_{2}-\omega_{1}=\omega_{21} .
$$

Going to the higher-order approximations in the iterative procedure (62), we see that, in addition to the standard resonance conditions as $\omega=\omega_{21}$ or $\omega_{i}=\omega_{21}$, there appear the conditions of harmonic generation

$$
k \omega=\omega_{21} \quad(k=2,3, \ldots),
$$

when there is the sole alternating field, or the conditions of parametric conversion

$$
\sum_{j}\left( \pm \omega_{j}\right)=\omega_{21}
$$

if several alternating fields modulate the trapping potential.

The prediction of nonlinear harmonic generation using the averaging method is in excellent agreement with a respective numerical simulation for a harmonic potential of the form $U_{z}(z)=m_{0} \omega_{z}^{2} z^{2} / 2$, with $\omega_{z}=600 / \mathrm{s}$. To demonstrate harmonic generation we employed a harmonic driving field of the form (6) with $V_{1}(z)=\beta z^{2}$ and driving frequency $\omega_{1}=\left(E_{2}-E_{1}\right) /(2 \hbar) \approx 552 / \mathrm{s}$. For a sufficiently strong driving field $\left(\beta=1.1 \times 10^{-32} \mathrm{~J} / \mu \mathrm{m}^{2}\right.$, roughly half the strength of the trapping potential) we observed a strongly enhanced population of the second excited mode (excitation of the first excited state is forbidden by parity conservation). The result for the time averaged coefficients is shown in Fig. 10] and demonstrates a population of up to $30 \%$ in this mode, far more than is to be expected in absence of interaction. As in the case of an anharmonic driving field (see above) we also find a substantial (30 \%) excitation of other modes for a strong driving field.

The terms harmonic generation and parametric conversion are used here by analogy with nonlinear optics, where there exist analogous phenomena 45]. Similar effects occur as well for elementary excitations of Bose-Einstein condensates [48, 49, 50]. As we have shown, such effects may also arise for topological coherent modes.

In conclusion, the resonant generation of topological modes seems to provide a feasible mechanism for creating novel states of trapped Bose gases, containing the combinations of several such modes. Here, we have considered the resonant generation realized by modulating the trapping potential. Note that another possibility of imposing resonant perturbations could be done by inducing periodic variations in the atomic scattering length [51, 52. The latter case requires that the atoms are in a state close to a Feshbach resonance.

As we mentioned in the Introduction, a dipole topological mode was successfully generated in experiment [23]. The resonant method of vortex creation was analysed in detail in [9]-11. Thus, the possibility of generating a single topological mode is well justified. The mode can be of any nature, whether dipole or vortex. The method of resonant generation works for any mode. Our numerical simulations for the GPE, with realistic physical parameters, show that the simultaneous generation of several topological modes is also feasible.

Acknowledgement: This work was supported by the Heisenberg-Landau Program, Alberta's informatics Circle of Research Excellence (iCORE), the Forschergruppe Quantengase, and the Optik Zentrum Konstanz. V.I.Y. wishes to thank Jürgen Audretsch for kind hospitality during his stay in Konstanz. 


\section{A Appendix. Description of numerical algorithms}

Our numerical simulations are performed for a BEC that obeys the GPE with an external potential of the form $U(\mathbf{r})=U_{\perp}\left(\mathbf{r}_{\perp}\right)+U_{z}(z)$, i.e., a transverse and a longitudinal trapping potential. We consider a quasi one-dimensional situation for which the energy required to create excited states of the transverse trapping potential $U_{\perp}\left(\mathbf{r}_{\perp}\right)$ is much higher than any other energy. The wavefunction then takes the approximate form $\varphi(\mathbf{r}, t)=\varphi_{\perp}\left(\mathbf{r}_{\perp}\right) \psi(z, t)$, where $\varphi_{\perp}\left(\mathbf{r}_{\perp}\right)$ is the transverse ground state. By integrating over the transverse coordinates $\mathbf{r}_{\perp}$ one can show that $\psi$ fulfills a one-dimensional GPE of the form

$$
i \hbar \frac{\partial \psi}{\partial t}=\left(\frac{\hbar^{2}}{2 m_{0}} \frac{\partial^{2}}{\partial z^{2}}+U_{z}(z)+N A_{z}|\psi|^{2}+\hat{V}\right) \psi,
$$

with an effective coupling parameter $A_{z} \equiv A_{s} \int\left|\varphi_{\perp}\left(\mathbf{r}_{\perp}\right)\right|^{4} d \mathbf{r}_{\perp}$, whereby the integral over the transverse ground state is of the order of the transverse width squared. The details of this reduction can be found in review [2].

In this appendix we shall present the methods used for a direct numerical solution of the one-dimensional GPE for different situations discussed in the preceding sections. We generally consider a BEC of $1000{ }^{87} \mathrm{Rb}$ atoms $\left(m_{0}=1.45 \times 10^{-25} \mathrm{~kg}, a_{s}=5.4 \mathrm{~nm}\right)$ with a transverse width of $7 \mu \mathrm{m}$. Our 1D simulation described a time evolution of $500 \mathrm{~ms}$ using $10^{7}$ discrete time steps. It was performed on a grid of 512 spatial points extending over a range of $26 \mu \mathrm{m}$.

\section{A.1 Numerical simulation of time evolution and determination of nonlinear coherent modes}

To simulate the time evolution governed by Eq. (76) we have employed the well-known split-step Fourier method [53. As initial condition we used the ground-state nonlinear coherent mode $\varphi_{0}(z)$ associated with the potential $U_{z}(z)$ in absence of a driving field $V(z, t)$. This ground state as well as higher nonlinear coherent modes were numerically determined by an imaginary-time propagation followed by a self-consistent field (SCF) method.

The imaginary-time propagation was described, for instance, in Ref. [54]. Very roughly speaking it is a method to obtain a kind of a small-temperature (corresponding to large imaginary time) collective wavefunction which approaches the ground state wavefunction for very large times. It usually gives excellent results for the ground state but fails to reproduce excited nonlinear coherent modes unless certain symmetry requirements are fulfilled. We used this method for intermediate imaginary times to obtain a better trial wavefunction $\varphi^{(0)}$ for the following SCF procedure. This step is not necessary but helps to improve the speed of the numerical algorithm.

The SCF method consists in inserting the trial wavefunction $\varphi^{(0)}$ into the nonlinear interaction part of Eq. (76). It thus provides a kind of potential term and turns Eq. (76) into a linear equation for $\psi$. We then solved for the stationary eigenstates of this equation. One of these states is then chosen to become a new trial wavefunction $\varphi^{(1)}$ and then the procedure is iterated until the overlap between $\varphi^{(n)}$ and $\varphi^{(n+1)}$ deviates by less than $\varepsilon$ from 1 (we have chosen $\varepsilon=10^{-14}$ ). The choice of the appropriate new trial 
wavefunction depends on the problem at hand. In the case of a 1D Schrödinger equation with a confining potential, the eigenstates are real and the number $N_{0}$ of zeros of each nonlinear coherent mode turned out to be a good criterion for the next trial wavefunction. To determine the ground state we picked the $N_{0}=0$ eigenstate in each iteration. For the excited coherent modes $N_{0}=1$ and 2 was the respective choice.

The convergence of the SCF method is the slower the higher the excited state one is looking for, and it even can fail to converge for $N_{0} \geq 2$. We therefore implemented a convergence acceleration scheme based on Anderson mixing as described in Ref. [55. In short, this scheme enhances the usual SCF algorithm by adding a kind of memory in the sense that $\varphi^{(n+1)}$ may depend not only on $\varphi^{(n)}$ but on previous trial wavefunctions, too. In practice, this memory lasts for 3-6 iterations. To implement this scheme one first writes

$\varphi^{(n+1)}$ as a superposition of the previous trial wavefunctions. Then one minimizes the deviation of this function from the previous trial wavefunction with respect to the superposition coefficients. This leads to a simple linear system of equations for the coefficients of the superposition. Anderson mixing can speed up convergence by orders of magnitude and turned out to be very suitable for our case. The three nonlinear coherent modes of lowest energy for a harmonic potential and an anharmonic potential are displayed in Fig. 3.

We have checked if the nonlinear coherent modes obtained in this way are indeed stationary solutions of the nonlinear Schrödinger equation by propagating them for a sufficiently long time using the split step algorithm. All modes were found to be perfectly stationary: their density does not change and their phase remains spatially homogeneous apart from the sharp phase jump by $\pi$ that appears at the zeros of the excited states, and apart from areas with extremely low densities (less than about $10^{-13}$ of the peak density) where numerical errors lead to a strongly fluctuating phase.

\section{A.2 Comparison to averaging procedure}

To compare the result of the direct numerical simulation with that obtained by the averaging procedure we first projected the numerical solution $\psi(z, t)$ found using the split-step method on the respective nonlinear coherent mode to obtain as an intermediate result the non-averaged coefficients $\tilde{c}_{i}\left(t_{l}\right):=\left(\varphi_{i}, \psi\left(t_{l}\right)\right) \exp \left(i E_{i} t_{l} / \hbar\right)$ with $t_{l}=l \Delta t, l=0,1,2, \ldots$. Since the nonlinear coherent modes $\varphi_{i}$ are not orthogonal the sum $\sum_{i}\left|\tilde{c}_{i}\left(t_{l}\right)\right|^{2}$ is not unity and the individual coefficients are oscillating on short time scales.

To obtain the coefficients $c_{i}\left(t_{l}\right)$ corresponding to the averaging procedure we stored the wavefunction at a total number of $N_{t}$ time steps $\left(N_{t}\right.$ was typically on the order of 1000) and defined the averaged coefficients as

$$
c_{i}\left(T_{n}\right):=\frac{1}{N_{a}} \sum_{l=1}^{N_{a}} \tilde{c}_{i}\left(T_{n}+t_{l}\right),
$$

where $N_{a}$ is the number of steps we averaged over and $T_{n}:=N_{a} n \Delta t, n=0,1,2, \ldots$. Generally $N_{a}=10$ to $N_{a}=32$ resulted in a fairly smooth time evolution of the coefficients $c_{i}(t)$ which can directly be compared to the results of the averaging procedure. 


\section{References}

[1] A.S. Parkins and D.F. Walls, Phys. Rep. 303, 1 (1998).

[2] P.W. Courteille, V.S. Bagnato, and V.I. Yukalov, Laser Phys. 11, 659 (2001).

[3] A.L. Fetter, J. Low Temp. Phys. 129, 263 (2002).

[4] S. Stenholm, Phys. Scr. T 102, 89 (2002).

[5] L.P. Pitaesvkii and S. Stringari, Bose-Einstein Condensation (Oxford University, Oxford, 2003).

[6] V.I. Yukalov, E.P. Yukalova, and V.S. Bagnato, Phys. Rev. A 56, 4845 (1997).

[7] V.I. Yukalov, Statistical Green's Functions (Queen's University, Kingston, 1998).

[8] V.I. Yukalov, E.P. Yukalova, and V.S. Bagnato, Phys. Rev. A 66, 043602 (2002).

[9] K.P. Marzlin, W. Zhang, and E.M. Wright, Phys. Rev. Lett. 79, 4728 (1997).

[10] K.P. Marzlin and W. Zhang, Phys. Rev. A 57, 3801 (1998).

[11] K.P. Marzlin and W. Zhang, Phys. Rev. A 57, 4761 (1998).

[12] E.A. Ostrovskaya, Y.S. Kivshar, M. Lisak, B. Hall, F. Cattani, and D. Anderson, Phys. Rev. A 61, 031601 (2000).

[13] D.L. Feder, M.S. Pindzola, L.A. Collins, B.I. Schneider, and C.W. Clark, Phys. Rev. A 62, 053606 (2000).

[14] V.I. Yukalov, E.P. Yukalova, and V.S. Bagnato, Laser Phys. 10, 26 (2000).

[15] Y.S. Kivshar, T.J. Alexander, and S.K. Turitsyn, Phys. Lett. A 278, 225 (2001).

[16] B. Damski, Z.P. Karkuszewski, K. Sacha, and J. Zakrzewski, Phys. Rev. A 65, 013604 (2002).

[17] V.I. Yukalov, E.P. Yukalova, and V.S. Bagnato, Laser Phys. 11, 455 (2001).

[18] R. D'Agosta, B.A. Malomed, and C. Presilla, Laser Phys. 12, 37 (2002).

[19] R. D'Agosta and C. Presilla, Phys. Rev. A 65, 043609 (2002).

[20] N.P. Proukakis and P. Lambropoulos, Eur. Phys. J. D 19, 355 (2002).

[21] V.I. Yukalov, E.P. Yukalova, and V.S. Bagnato, Laser Phys. 12, 231 (2002).

[22] V.I. Yukalov and E.P. Yukalova, J. Phys. A 35, 8603 (2002).

[23] J. Williams, R. Walser, J. Cooper, E.A. Cornell, and M. Holland, Phys. Rev. A 61, $033612(2000)$. 
[24] G.J. Milburn, J. Corney, E.M. Wright, and D.F. Walls, Phys. Rev. A 55, 4318 (1997).

[25] S. Raghavan, A. Smerzi, S. Fantoni, and S.R. Shenoy, Phys. Rev. A 59, 620 (1999).

[26] R. Franzosi and V. Penna, Laser Phys. 12, 71 (2002).

[27] A. Bhattacherjee and M. Mohan, Mod. Phys. Lett. B 16, 1021 (2002).

[28] A.J. Leggett, Rev. Mod. Phys. 47, 331 (1975).

[29] P. Ohberg and S. Stenholm, Phys. Rev. A 59, 3890 (1999).

[30] U.V. Poulsen and K. Mølmer, Phys. Rev. A 64, 013616 (2001).

[31] B. Wu and Q. Niu, Phys. Rev. A 61, 023402 (2000).

[32] B. Wu, R.B. Diener, and Q. Niu, Phys. Rev. A 65, 025601 (2002).

[33] J. Liu, L. Fu, B.Y. Ou, S.G. Chen, D.I. Choi, B. Wu, and Q. Niu, Phys. Rev. A 66, 023404 (2002).

[34] M. Machholm, C.J. Pethick, and H. Smith, e-print cond-mat/0301012 (2003).

[35] V.I. Yukalov, E.P. Yukalova, and V.S. Bagnato, Laser Phys. 12, 1325 (2002).

[36] V.I. Yukalov, E.P. Yukalova, and V.S. Bagnato, Phys. Rev. A 66, 025602 (2002).

[37] J.J. Garcia-Ripoll, V.M. Pérez-Garcia, and P. Torres, Phys. Rev. Lett. 83, 1715 (1999).

[38] P.A. Ruprecht, M.J. Holland, K. Burnett, and M. Edwards, Phys. Rev. A 51, 4704 (1995).

[39] A. Gammal, L. Tomio, and T. Frederico, Phys. Rev. A 66, 043619 (2002).

[40] P.E. Zhidkov, Int. J. Mod. Phys. A 12, 295 (1997).

[41] P.E. Zhidkov, Korteweg - de Vries and Nonlinear Schrödinger Equations: Qualitative Theory (Springer, Heidelberg, 2001).

[42] P.E. Zhidkov, Nonlinear Anal. 52, 737 (2003).

[43] N.N. Bogolubov and Y.A. Mitropolsky, Asymptotic Methods in the Theory of Nonlinear Oscillations (Gordon and Breach, New York, 1961).

[44] L.D. Faddeev and L.A. Takhtajan, Hamiltonian Methods in the Theory of Solitons (Springer, Berlin, 1987).

[45] L. Mandel and E. Wolf, Optical Coherence and Quantum Optics (Cambridge University, Cambridge, 1995).

[46] V.I. Yukalov, Phys. Rev. B 53, 9232 (1996). 
[47] G. Joos and D.D. Joseph, Elementary Stability and Bifurcation Theory (Springer, New York, 1980).

[48] P.A. Ruprecht, M. Edwards, K. Burnett, and C.W. Clark, Phys. Rev. A 54, 4178 (1996).

[49] F. Dalfovo, C. Minniti, and L.P. Pitaevskii, Phys. Rev. A 56, 4855 (1997).

[50] G. Hechenblaikner, O.M. Marago, E. Hodby, J. Arlt, S. Hopkins, and C.J. Foot, Phys. Rev. Lett. 85, 692 (2000).

[51] S.K. Adhikari, Phys. Lett. A 308, 302 (2003).

[52] S.K. Adhikari, J. Phys. B 36, 1109 (2003).

[53] P.L. DeVries, AIP Conf. Proc. 160, 269 (1986).

[54] F. Dalfovo and S. Stringari, Phys. Rev. A 53, 2477 (1996).

[55] V. Eyert, J. Comp. Phys. 124, 271 (1996). 


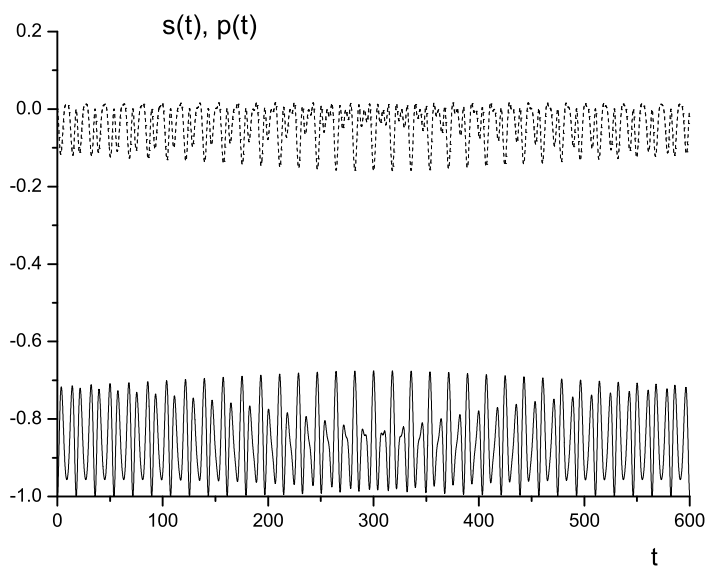

Figure 1: Mode locked regime for the case of three coupled nonlinear modes. Parameters are $\alpha_{i j}=\alpha, b_{i j}=0.35 \alpha$, and $\delta_{i}=0$. Initial conditions are $s_{0}=-1, p_{0}=0, x_{0}=y_{0}=0$. Time is measured in units of $\alpha^{-1}$. Shown are the population differences $s(t)$ (solid line) and $p(t)$ (dashed line).

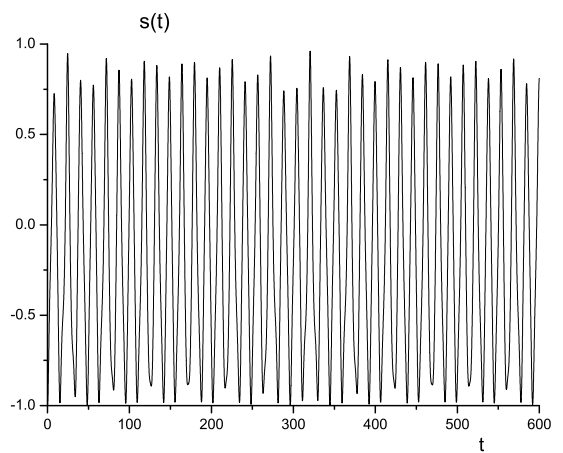

a)

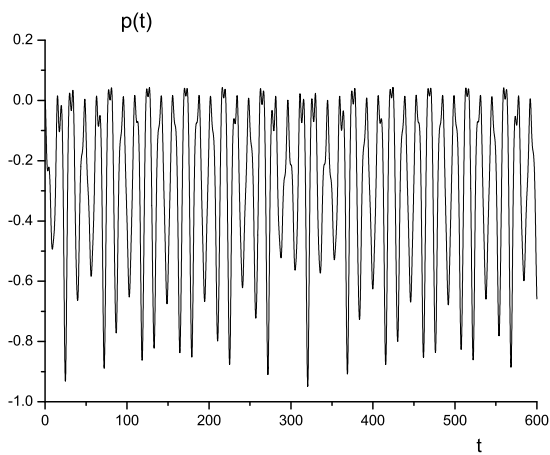

b)

Figure 2: Mode unlocked regime for the three-mode case. All parameters and initial conditions are the same as in Fig. 1 , except $b_{i j}=0.55 \alpha$. Again, time is shown in units of $\alpha^{-1}$. The population differences: (a) $s(t)$; (b) $p(t)$. 
a)
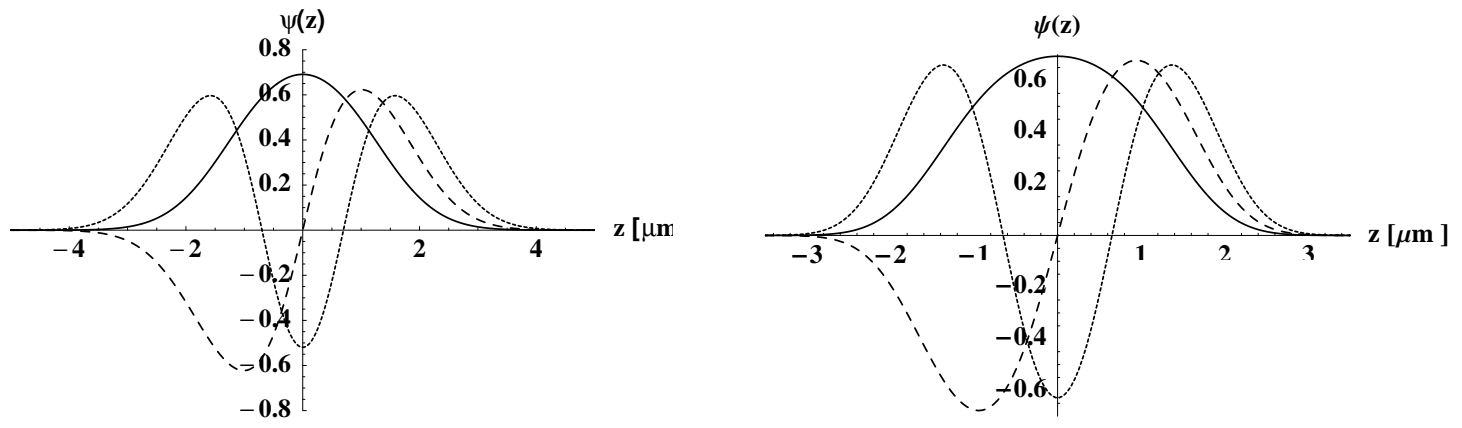

Figure 3: The three nonlinear coherent modes of lowest energy for $1000{ }^{87} \mathrm{Rb}$ atoms in two different trapping potentials. The solid line corresponds to the ground state, the dashed (dotted) line to the first (second) excited mode, respectively. a) harmonic trap with frequency $\left.\omega_{z}=600 / \mathrm{s}, \mathrm{b}\right)$ anharmonic trap of the form $U_{0} z^{4}$ with $U_{0}=10^{-32} \mathrm{~J} / \mu \mathrm{m}^{4}$.
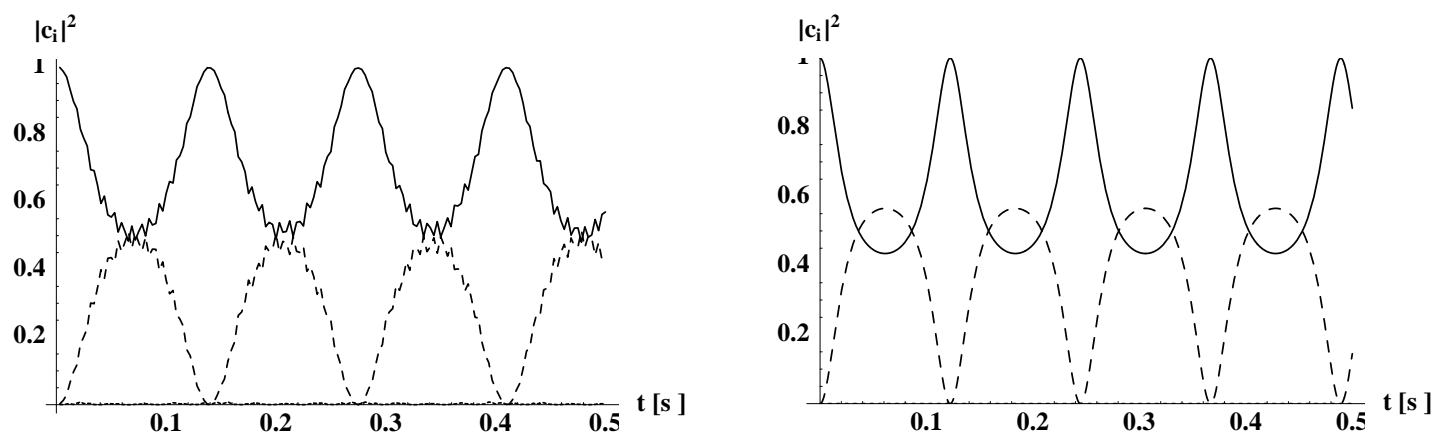

Figure 4: Mode coefficients averaged over a time of $3 \mathrm{~ms}$ for a BEC in an anharmonic trap $\propto z^{4}$ driven resonantly by a slightly subcritical linear potential. a) results of direct numerical simulation, b) predictions of the averaging method (see text for more details). Solid line: $\left|c_{0}(t)\right|^{2}$, dashed line: $\left|c_{1}(t)\right|^{2}$, dotted line: $\left|c_{2}(t)\right|^{2}$.
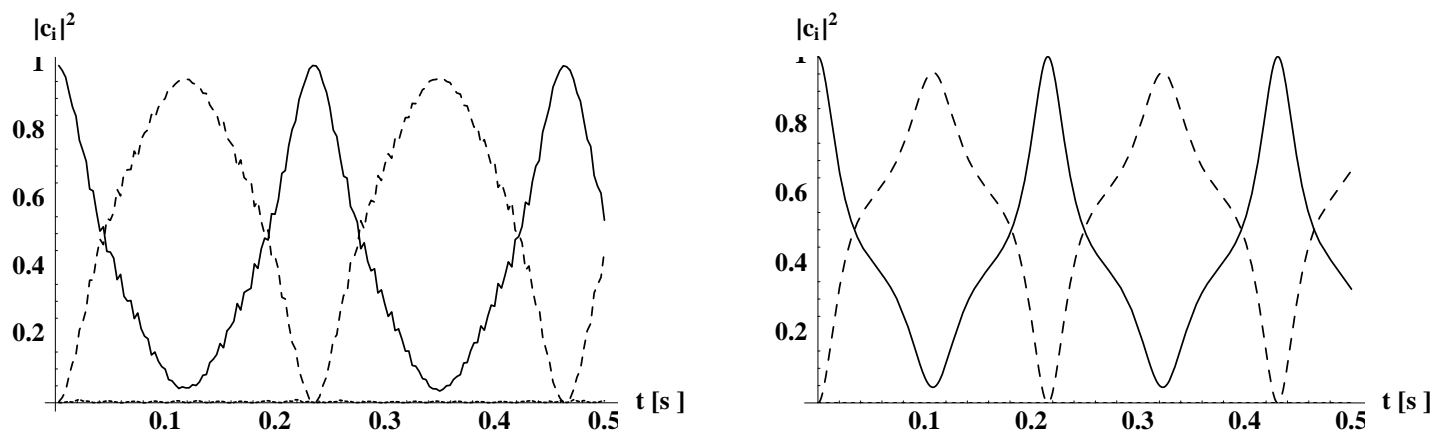

Figure 5: The same as Fig. 4, but for a slightly supercritical driving force. 

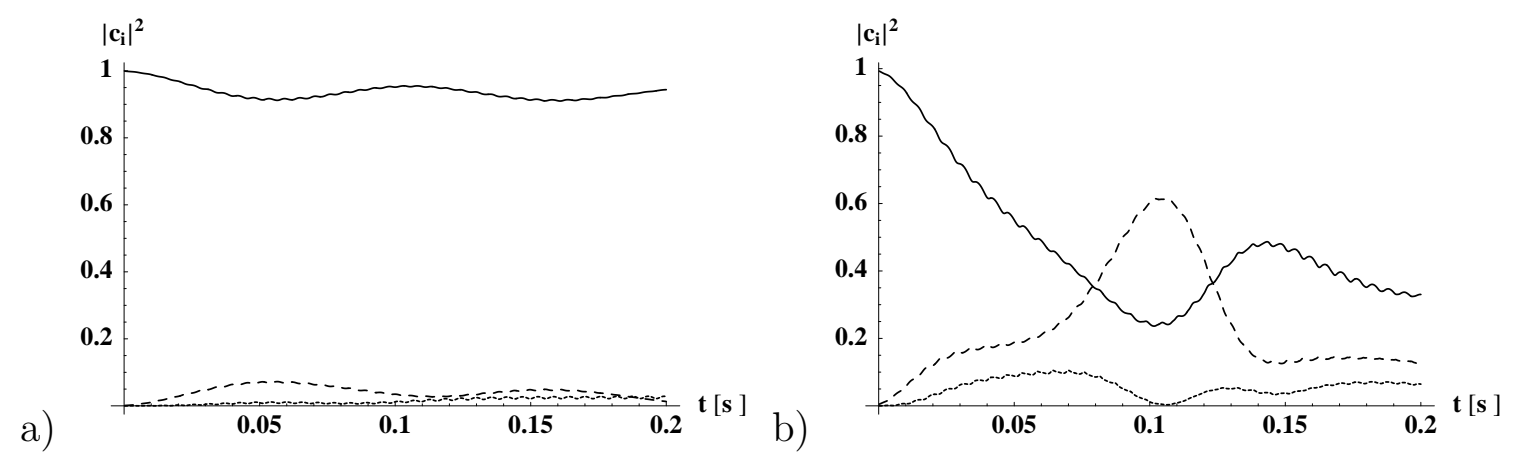

Figure 6: Mode coefficients averaged over a time of $5.3 \mathrm{~ms}$ for a BEC in a harmonic trap driven resonantly by a screened cubic potential. a) weak driving force with $\beta_{1}=$ $0.7869 \times 10^{-33} \mathrm{~J} / \mu \mathrm{m}^{3}$ and $\beta_{2}=0.344 \times 10^{-33} \mathrm{~J} / \mu \mathrm{m}^{3}$. b) strong driving force with $\beta_{1}=1.96734 \times 10^{-33} \mathrm{~J} / \mu \mathrm{m}^{3}$ and $\beta_{2}=0.859975 \times 10^{-33} \mathrm{~J} / \mu \mathrm{m}^{3}$. Solid line: $\left|c_{0}(t)\right|^{2}$, dashed line: $\left|c_{1}(t)\right|^{2}$, dotted line: $\left|c_{2}(t)\right|^{2}$.

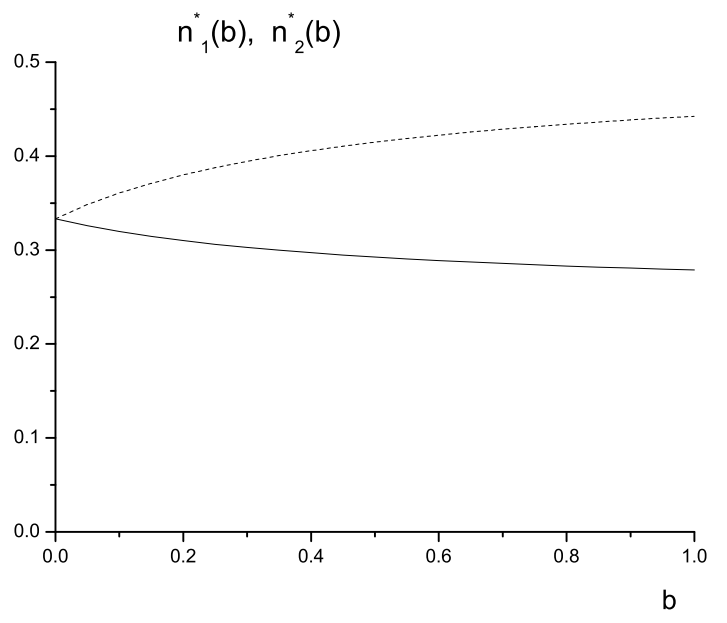

Figure 7: Stationary solutions for the case $x^{*}=y^{*}=0$ and $n_{1}^{*}=n_{3}^{*}$ as functions of the pumping parameter $b$ : stable $n_{1}^{*}(b)=n_{3}^{*}(b)$ (solid line), stable $n_{2}^{*}(b)$ (dashed line). 


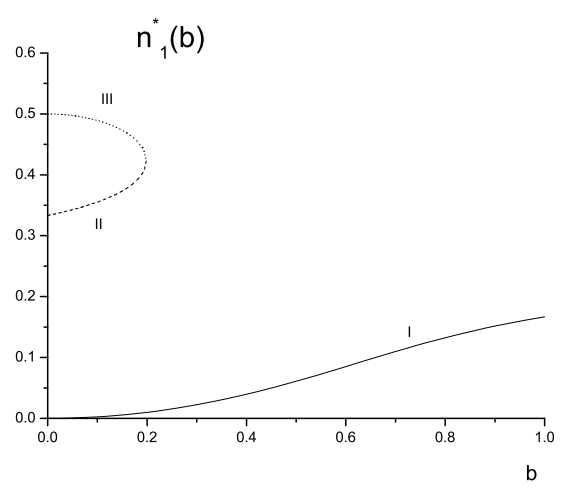

a)

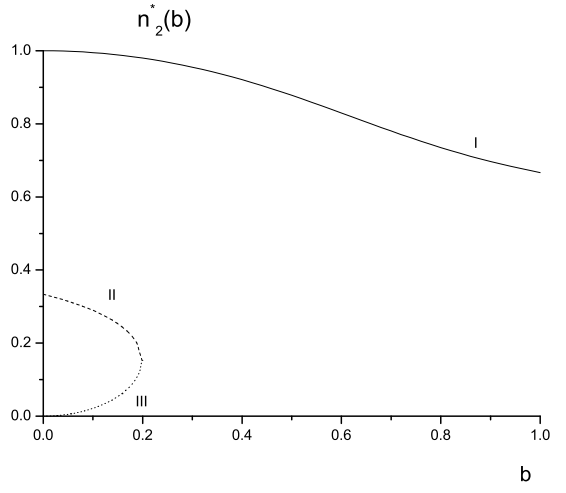

b)

Figure 8: Stationary solutions related to the fixed point $x^{*}=y^{*}=\pi$ and $n_{1}^{*}=n_{3}^{*}$ as functions of the pumping parameter $b$. Stable branch I (solid line), unstable branch II (dashed line), and unstable branch III (dashed line): (a) $n_{1}^{*}(b)=n_{3}^{*}(b) ;(\mathrm{b}) n_{2}^{*}(b)$.

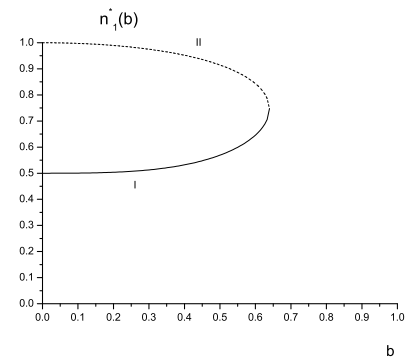

a)

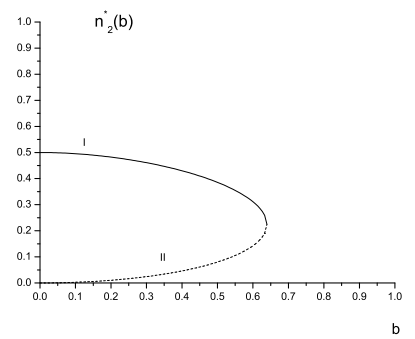

b)

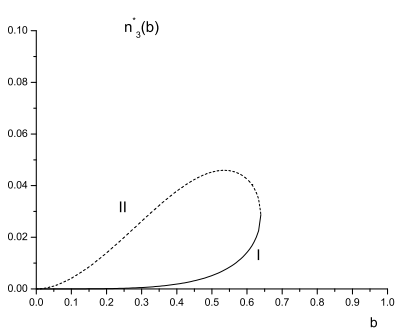

c)

Figure 9: Stationary solutions associated with the fixed point $x^{*}=y^{*}=\pi$ and $n_{1}^{*}>n_{3}^{*}$ as functions of $b$. Stable branch I (solid line) and unstable branch II (dashed line): (a) $n_{1}^{*}(b) ;(\mathrm{b}) n_{2}^{*}(b) ;(\mathrm{c}) n_{3}^{*}(b)$.

a)

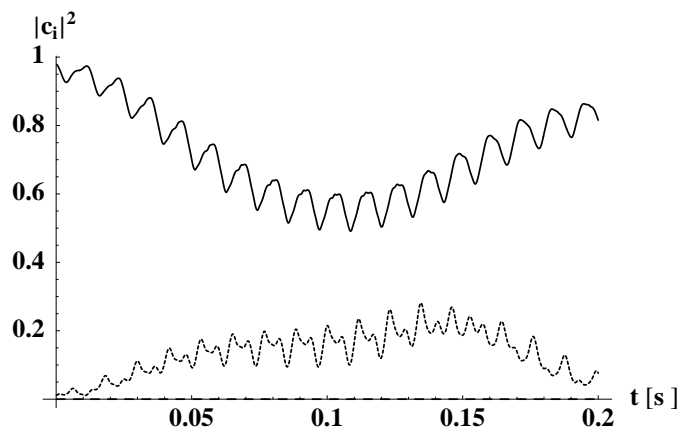

b)

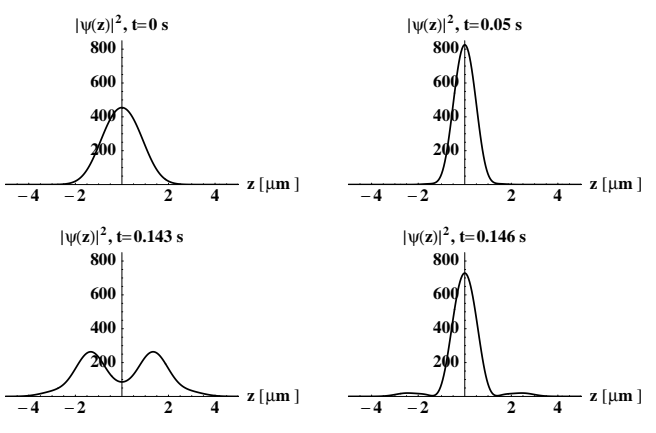

Figure 10: Time evolution of a BEC trapped and driven by harmonic potentials. a) mode coefficients averaged over a time of $5.3 \mathrm{~ms}$ (the lines have the same meaning as in Fig. 4), b) spatial probability density at different time steps. The effect of harmonic generation of population in the second excited state can clearly be seen in both figures. 